Cahiers de recherches médiévales

\title{
César est-il un personnage de roman?
}

Du Perceforest au Jouvencel

\section{Michelle Szkilnik}

\section{(2) OpenEdition \\ Journals}

\section{Édition électronique}

URL : https://journals.openedition.org/crm/849

DOI : $10.4000 / \mathrm{crm} .849$

ISSN : $1955-2424$

\section{Éditeur}

Honoré Champion

Édition imprimée

Date de publication : 30 mars 2006

Pagination : 77-89

ISSN : 1272-9752

\section{Référence électronique}

Michelle Szkilnik, "César est-il un personnage de roman? », Cahiers de recherches médiévales [En ligne], 13 spécial | 2006, mis en ligne le 03 avril 2009, consulté le 15 décembre 2022. URL : http:// journals.openedition.org/crm/849; DOI : https://doi.org/10.4000/crm.849 


\section{RM}

\section{César est-il un personnage de roman? Du Perceforest au Jouvencel}

César est-il un personnage de roman? F. Suard posait la même question au sujet d'un autre héros antique Alexandre, voilà plus de quinze ans ${ }^{1}$. Il y répondait par l'affirmative et ajoutait : «Le héros du Ralix n'est pas seulement un type historique dont les fautes auraient été gommées; il ne s'identifie pas non plus à une figure épique tournée d'abord vers des exploits guerriers. Sans négliger les relations étroites qui unissent le personnage et l'œuvre à l'histoire et à l'épopée, nous voyons Alexandre entrer, au prix d'une construction littéraire significative, dans l'orbite romanesque. $»^{2}$ Ce qui lui paraît faire d'Alexandre un personnage de roman, c'est " la cohérence d'une exemplarité universelle, et la rencontre de l'échec et de la mort, fruit d'une passion liée à cette exemplarité même. $»^{3}$

Qu'en est-il de César? Rappelons d'abord que si César est bien connu, il n'est pas devenu, à la différence d'Alexandre, le héros d'une grande fresque narrati$\mathrm{ve}^{4}$. Tout juste Jean de Thuin compose-t-il Li Roumanz de Julius Caesar au milieu du XIII ${ }^{\mathrm{e}}$ siècle. Mais, sous sa forme rimée ou sous sa version en prose, le texte n'aura pas le succès des divers romans d'Alexandre. Certes, dès le début du XIII ${ }^{\mathrm{e}}$ siècle, les Faits des Romains proposent une biographie du héros, mais fondée sur les textes de Salluste, Suétone et Lucain, elle se veut un texte historique, pas un roman 5 . Si César suscite l'enthousiasme, c'est plutôt, on le voit, comme personnage historique et historien lui-même. Figure exemplaire du conquérant, sans doute conduit à la mort par son ambition insatiable, à l'instar d'Alexandre, il n'a pourtant pas pu échapper, semble-t-il, à sa condition de personnage historique. Ou du moins qu'à de rares occasions.

Je voudrais examiner deux textes tardifs pour mettre en évidence d'une part le traitement «conventionnel» que subit César dans bon nombre de récits médiévaux, et d'autre part, la manière dont, dans un cas très particulier, il a été attiré dans le champ du romanesque. Le premier de ces textes est Le Jouvencel de Jean de Bueil, rédigé entre 1461 et 1468 ; et le second le Roman de Perceforest. On trouvera peut-être étrange et artificiel ce rapprochement, que je justifierai néanmoins de deux manières. D'une part, ces deux textes ont en commun d'être aux confins de l'histoire et du roman. D'autre part, ils sont, dans leur forme actuelle en tout cas, à peu près contemporains. La date du Perceforest reste en effet incertaine. Il est certes commu-

\footnotetext{
${ }^{1}$ F. Suard, «Alexandre est-il un personnage de roman? », Bien dire et bien aprandre, t. 7, 1989, p. 77-87.

${ }^{2}$ Art. cit. p. 86 .

${ }^{3}$ Art. cit. p. 81.

${ }^{4}$ Pour un panorama de l'utilisation du personnage au Moyen Âge, on se repportera au livre de J. Leeker, Die Darstellung Cäsars in den romanischen Literaturen des Mittelalters, Frankfurt am Main, V. Klostermann, 1986.

${ }^{5}$ Sur ce texte, ainsi que sur l'Histoire Ancienne jusqu'à César, voir C. Croizy-Naquet, Écrire l'histoire romaine au début du XIII siècle, Paris, Champion, 1999.
}

Cahiers de Recherches Médiévales, 13spé, 2006 
nément admis que le roman a été composé dans la première moitié du XIV ${ }^{\mathrm{e}}$ siècle. Mais son dernier éditeur, G. Roussineau, est revenu sur cette datation et a suggéré que le roman a sans doute subi une transformation radicale vers le milieu du $\mathrm{XV}^{\mathrm{e}}$ siècle. J'ai moi-même émis l'hypothèse que certains développements concernant César dans le Perceforest pourraient avoir été ajoutés à l'occasion de ce remaniement $^{6}$. En tout état de cause, les manuscrits qui conservent le roman datent tous de la seconde moitié du $\mathrm{XV}^{\mathrm{e} 7}$, c'est-à-dire qu'ils sont contemporains de ceux du Jouven$c e l$, et que le remaniement (s'il ne s'agit que d'un remaniement et non pas d'une rédaction pure et simple) est contemporain de la composition du récit de Jean de Bueil. Si on ajoute que l'un des manuscrits du Perceforest et l'un des manuscrits du Jouvencel ont été copiés pour le même mécène, Louis de Bruges, seigneur de la Gruthuyse $^{8}$, le rapprochement fait sens : un lecteur au moins, Louis de Bruges, a disposé de deux ouvrages donnant de César des images sensiblement différentes et pourtant complémentaires.

Le traictié narratif de Jean de Bueil, pour reprendre l'expression par laquelle il désigne son œuvre, est un récit à portée didactique mais dont la dimension romanesque me semble également indéniable". Toutefois César, conformément à l'usage qui est fait du personnage dans les ouvrages didactiques, n'y apparaît qu'à titre d'exemple dans les discours édifiants dont on abreuve le jeune héros. La première fois (chapitre V, première partie), c'est dans celui d'un vieux compagnon du Jouvencel qui cherche à convaincre le jeune homme d'embrasser pleinement la carrière des armes et surtout de renoncer à son idée de se rendre à la cour. Organisé en trois points le discours passe d'abord en revue les inconvénients de la vie de cour, puis la valeur de la vie militaire ${ }^{10}$. L'argument principal du vieux soldat est que seul l'exercice de la guerre peut conduire aux plus hauts honneurs :

\footnotetext{
${ }^{6}$ Voir mon article, «David Aubert chroniqueur, le prologue du Perceforest dans la compilation de l'Arsenal ", Seuils de l'œuvre dans le texte médiéval, éd. E. Baumgartner et L. Harf, Paris, Presses de la Sorbonne Nouvelle, 2002, p. 201-221.

${ }^{7}$ La copie de David Aubert qui conserve l'intégralité du roman (Paris, Ars. 3483-3494 ) date de 1459-1460. Les autres manuscrits (Paris, BNF français 345-348 et BNF français 106-109) datent respectivement de 1470-1475 et 1471-1477. Voir la présentation des manuscrits dans la quatrième partie de Perceforest, éd. G. Roussineau, t. 1, Genève, Droz, 1987, p. XXI-XXXI. Le manuscrit de Londres n'étant que la grosse de celui de David Aubert ne présente pas d'intérêt dans notre optique.

${ }^{8}$ Il s'agit pour le Perceforest des mss BNF français 345-348 et pour le Jouvencel du ms BNF français 192. Signalons en outre que le fils de Louis de Bruges, Jean, qui devait hériter de la magnifique bibliothèque de son père, avait épousé en 1480 Renée de Bueil, petite fille de Jean de Bueil.

${ }^{9}$ Sur ce point, voir mon article, «Figure exemplaire et personnage de roman : le Jouvencel de Jean de Bueil », à paraître dans les actes du colloque Vérité poétique, vérité politique, mythes, modèles et idéologies politiques au Moyen Âge, qui s'est tenu à la Faculté Victor-Segalen à Brest les 22-24 septembre 2005.

${ }^{10} \mathrm{Ce}$ point aborde «les hautes vertus, les grans triumphes, la loyaulté et grant couraige des gens de guerre»(Jouvencel, ms Escorial, fol. 19v). Je cite le texte dans le manuscrit de l'Escorial (Madrid, Escorial, S-11-16), à partir duquel je prépare une nouvelle édition du Jouvencel. Il existe une édition ancienne, celle de C. Favre et L. Lecestre (Société de l'Histoire de France, Paris, 1887-1889, 2 volumes).
} 
«Tout empire et toute seigneurie a prins commancement de guerre (Jouvencel, ms Escorial, fol. $19^{v}$ ).

«Tout l'onneur du monde est venu par conquerir et guerroier et jamais on ne conquiert sans faire guerre. »(Jouvencel, ms Escorial, fol. $\left.20^{\mathrm{v}}\right)$.

Le destin de César illustre ces déclarations : victorieux en Gaule, César revenait à Rome recevoir les honneurs qui lui étaient légitimement dus, mais Pompée poussa les Romains à lui refuser le triomphe :

J'ay oy reciter aux clers que anciennement la seigneurie des Rommains estoit renouvellee par chacun an et baillee a deux consulz, mais aprés que Jules Cesar retourna des parties de France et d'oultre les Alpes, les Rommains a l'admonestement du grant Pompee lui refuserent les honneurs et les triumphes appartenans a luy a cause des belles conquestes qu'il avoit faictes, dont il leur print si malement que pour ceste cause en fut faitte grande et horrible destruction es champs de Thessale. Si n'est point memoire que, puis ceste bataille merveilleuse qui fut entre luy et Pompee son gendre, advint ou siecle aussi grant effusion de sang ne aussi terrible desconfiture du lignaige humain pour une foiz. Et en mesprint si malement au dit Pompee pource qu'il avoit suggeré ce reffuz qu'il en perdit non pas seulement les grans honneurs et la preeminence qu'il avoit a Romme mais aussi sa vie piteusement. Et a cause de ceste victoire Jules Cesar osta le consulat de Romme et en fist empire et en fust empereur tout son vivant. (Jouvencel, ms. Escorial, fol. 20v)

L'attitude de Pompée apparaît comme injuste et catastrophique puisqu'elle conduit à la bataille de Pharsale, à la mort d'un grand nombre de Romains et à sa propre défaite. En revanche, la victoire de César et la prise de pouvoir qui s'ensuit sont présentées comme naturelles et justifiées. Aux yeux du vieux compagnon, l'accession au pouvoir de César, au terme d'une guerre de conquête et d'une guerre civile, n'a rien d'un coup d'état scandaleux. La responsabilité de la guerre civile et de l'effusion de sang qu'elle a provoquée est rejetée sur Pompée qui tout grand qu'il ait été endure la déconfiture qu'il a méritée. Et le vieux compagnon peut logiquement conclure de l'exemple:

Ainsi appert que par la guerre maintenir et suivir quant mestier en est, on parvient a ung treshault honneur et acquiert on les grans triumphes et les seigneuries. (Jouvencel, ms. Escorial, fol. $20^{\mathrm{v}}$ ).

Cette image positive de César est néanmoins considérablement altérée par la suite. En effet, le vieux capitaine de Crathor, qui est une sorte de mentor pour le Jouvencel, entend donner à celui-ci quelques principes moraux et nuance l'idée que toute guerre est nécessairement source d'honneurs : seule celle qui est entreprise «en bonne querelle» plaît à Dieu et est couronnée de succès. La guerre menée par orgueil, par envie, ou par avarice conduit «a confusion totale et deshonneur» 
(Jouvencel, ms Escorial, fol. $\left.50^{\mathrm{r}}\right)^{11}$. À l'appui de cette déclaration, le capitaine cite l'exemple d'Alexandre, puis celui de César :

Nous lisons aussi d'Alixandre le grant qui conquesta et subjuga tout le monde ; mais aprés il fut empoisonné par ung de ses familliers et n'eut aucun enfant ou hoir de son corps a qui sa conqueste proufitast. Julles Cesar aussi qui, selon l'oppinion des saiges, vainquit et subjuga le vertueux Pompee plus par orgueil et advenement de fortune que de justice ou equalité de guerre, fut en la fin murtry ou Capitolle par les greffes de Brutus et d'autres senateurs. Pource doivent les gens de guerre ensuivir a leur povoir bonnes querelles et ce qu'ilz entreprennent le doivent excecuter en grant humilité et soubz la crainte de Dieu. (Jouvencel, ms Escorial, fol. 51 ${ }^{v}$ ).

La présentation de la guerre civile et de ses protagonistes est bien différente de ce qu'elle était dans le discours du vieux compagnon : le grand Pompée devient le vertueux Pompée, la victoire de César n'apparaît plus comme légitime ni même comme logique. C'est un coup du sort, une ironie de Fortune, qui permet à César de l'emporter. Mais Dieu, ou Fortune, rétablit la justice en faisant tomber l'empereur sous les greffes des sénateurs romains, de même qu'il a autorisé l'empoisonnement d'Alexandre.

Exalté comme un modèle positif dans le discours du vieux compagnon, César devient dans celui du capitaine l'exemple à ne surtout pas suivre. Ce renversement témoigne soit de la plasticité du personnage, soit plutôt du malaise que suscite cet homme de guerre dont on admire indiscutablement les conquêtes et les hauts faits militaires mais sur les qualités morales de qui on s'interroge sans doute aussi.

La troisième allusion à César dans le Jouvencel inverse de nouveau la perspective. C'est cette fois un envoyé du roi, maistre Nicolle, qui s'adresse au Jouvencel au moment où celui-ci est promu lieutenant du roi à Crathor. Son long discours moral a pour objet de rappeler au futur lieutenant les devoirs du chevalier et la nécessité de ne s'engager dans la guerre qu' «a bon droit et a bonne cause » (Jouvencel, ms Escorial, fol. $\left.131^{\mathrm{r}}\right)$. Cette préoccupation fait écho aux propos tenus préalablement par le capitaine de Crathor. Il est donc surprenant de retrouver l'exemple de César utilisé de manière positive dans un développement qui recommande la miséricorde aux chefs de guerre, qualité dont César a su faire preuve :

Aussi devez savoir qu'il n'est riens plus necessaire a ung bon prince ou chevalier que estre piteux et enclin a misericorde. A ce propos racompte Valere en son huitiesme livre comme Marcellinus print la cité de Ciracuse, mais quant il vit les prisonniers, femmes et enffans, lesquelz plouroient, se prist a plourer. En ce mesme livre lisons que Julius Cesar voyant la teste de Pompeyus son ennemy mortel fut moult couroucé et eut grant pitié. (Jouvencel, ms Escorial, fol. 132 ${ }^{\mathrm{r}}$ ).

\footnotetext{
${ }^{11}$ «Et s'il advient par aucune aventure qu'ilz (= ceux qui sont mus par l'orgueil) parviennent a l'acomplissement de leur desir et qu'ilz soient hault eslevez et essaulcez en grant estat, neantmoins tousjours leur advient il quelque esclandre a la parfin. » (Jouvencel, ms Escorial, fol. $\left.51^{r}\right)$
} 
On dira que maistre Nicolle ne s'interroge pas ici sur la légitimité des guerres entreprises par César, mais se contente de louer sa réaction de pitié devant le corps de son ennemi. Il n'en demeure pas moins que c'est bien César qui est à l'origine de la déroute de Pompée et indirectement de sa mort. Les larmes qu'il verse sur son ennemi le laveraient-elles de sa responsabilité?

En tout état de cause la manière dont le Jouvencel utilise le personnage de César appelle plusieurs remarques. D'abord, je l'ai dit, César n'apparaît jamais qu'à titre d'exemple historique, dans le discours de personnages. Ensuite, il est souvent couplé avec Alexandre, ce qui n'a rien d'étonnant, compte tenu que les deux héros figurent depuis Jacques de Longuyon au rang des trois preux antiques dans le panthéon des neuf preux. Enfin, comme Alexandre, César est susceptible de fournir un exemple positif ou un exemple négatif, au gré des besoins de l'argumentation. Bien que le Jouvencel soit en bonne partie un récit contant les aventures fictives d'un jeune chevalier, l'usage qu'il fait de César est comparable à celui qu'en font les oeuvres didactiques, historiques ${ }^{12}$ ou poétiques ${ }^{13} \mathrm{du} \mathrm{XV}^{\mathrm{e}}$ siècle. César est essentiellement un personnage historique dont la destinée fournit de bonnes leçons au bénéfice des hommes de guerre de la fin du Moyen Âge. Alors qu'Alexandre échappe à l'Histoire pour accéder au rang de personnage de fiction et devenir le héros de longues œuvres romanesques ${ }^{14}$, César aux $\mathrm{XIV}^{\mathrm{e}}$ et $\mathrm{XV}^{\mathrm{e}}$ siècles reste le plus souvent cantonné dans son rôle de modèle historique.

Excepté dans mon deuxième texte, le Roman de Perceforest, auquel je voudrais passer à présent. Perceforest est un énorme roman qui s'attache à combiner l'histoire d'Alexandre et celle du royaume arthurien, en faisant d'Alexandre et de ses deux lieutenants Bétis et Gadiffer les ancêtres des chevaliers arthuriens. Remarquons donc d'emblée que le héros antique qui aura la vedette, c'est encore une fois Alexandre et non César. Pourtant ce dernier joue un rôle qui est loin d'être négligeable et qui révèle ce qui m'apparaît bien comme une véritable mise en roman du

${ }^{12}$ Dans son Voyage d'Oultremer (voyage réalisé en 1432, bien que la rédaction en soit plus tardive), Bertrandon de la Broquiere assure être passé près du lieu où s'est déroulée «la bataille de Thessale de Jule Cesar et de Pompee » (éd. Ch. Schefer, Paris, Leroux, 1892, reprint Gregg International Publishers Limited, Westmead, Farnborough, Hants, England, 1972, p. 172 et p. 178). L'allusion fonctionne ici comme garantie du sérieux géographique et historique de la relation de voyage. Notons que comme le Jouvencel, le Voyage parle de la bataille à Thessale et non de Pharsale. Autre allusion à César p. 219.

${ }^{13}$ Dans Le Chevalier Délibéré d'Olivier de la Marche par exemple, l'ermite Entendement montre à l'Acteur les greffes qui ont servi à tuer César et lui explique que cette mort pitoyable est l'œuvre de Messire Accident. Puis il lui fait voir la boite qui contenait le poison qu'Antipater a porté à Alexandre (strophes 55 et 56, éd. C. W. Carroll, Tempe, Arizona, Arizona Center for Medieval and Renaissance Studies, 1999). Dans la Ballade de Fortune de Villon, Fortune se vante de même d'avoir trahi Jules César au sénat, perdu Pompée en Egypte et empoisonné Alexandre (voir éd. J.Cl. Mühlethaler, Lais, Testament, Poésies diverse, Paris, Champion, 2004, vv. 2079, 2080 et 2084).

${ }^{14}$ Pour le XIV ${ }^{\mathrm{e}}$ siècle, on citera les Væux du Paon de Jacques de Longuyon, ainsi que ses suites, Le Restor du Paon de Jean le Court, dit Brisebarre, et Le Parfait du Paon de Jean de le Mote ; pour le $\mathrm{XV}^{\mathrm{e}}$ le Roman d'Alexandre de Jean Wauquelin, ainsi que la rédaction anonyme en prose contenue dans le manuscrit de Besançon. 
personnage. Conformément à sa stature historique, c'est toutefois en qualité de chef de guerre et d'homme politique qu'il apparaît. Dans le Perceforest, César est uniquement un conquérant, celui qui débarque en Grande-Bretagne dans l'espoir d'ajouter ce royaume aux terres qu'il a déjà soumises sur le continent. En ce sens, son action est beaucoup plus spécifique et limitée que celle d'Alexandre, qui pour sa part ne cherche pas à annexer la Grande-Bretagne. Jeté par une tempête sur les côtes d'Angleterre en compagnie de quelques hommes, Alexandre découvre un pays désolé, sans roi ni loi. Il propose alors à la population qui l'a recueilli sur le rivage de lui donner pour nouveau seigneur l'un de ses lieutenants, le jeune Bétis, futur Perceforest. Cette proposition est acceptée avec joie. Le début du roman raconte la pacification de la Grande-Bretagne (Angleterre et Ecosse) et la mise en place d'institutions justes et bénéfiques pour les habitants de l'île. Si conquête il y a eu, c'est une conquête paisible qui apporte la stabilité et la prospérité à un pays meurtri par les exactions du mauvais lignage de l'enchanteur Darnant. Alexandre non seulement ramène la paix mais encore instaure les coutumes courtoises qui perdureront jusqu'à l'époque d'Arthur'15.

Il en va tout autrement des entreprises de César qui chaque fois sèment la ruine et la désolation en Grande-Bretagne. Le rôle de conquérant brutal est sans doute en partie imposé à César par les textes arthuriens des $\mathrm{XII}^{\mathrm{e}}$ et $\mathrm{XIII}^{\mathrm{e}}$ siècles. En effet, chez Wace, les Romains sont présentés comme les ennemis quasi héréditaires d'Arthur, toujours prêts à envahir la Grande-Bretagne. Les romans en prose du XIII ${ }^{\mathrm{e}}$ siècle, du Lancelot aux Prophésies de Merlin, entretiennent l'histoire de cette lutte séculaire entre les deux peuples. Le Perceforest, racontant la préhistoire du monde arthurien, reprend le motif pour relater l'origine de la guerre et décrire une première expédition des Romains, avant même celle de César, qui s'était soldée par un cuisant échec pour les envahisseurs ${ }^{16}$. Quoi de plus naturel alors que de faire appel au plus fameux des Romains pour venger l'affront, et de dresser les deux grands héros antiques, Alexandre et César, l'un contre l'autre, même au prix d'une sévère distorsion temporelle? ${ }^{17}$

En vérité, César n'affronte pas directement Alexandre mais ses amis, les rois Perceforest de Grande-Bretagne et Gadiffer d'Ecosse. Dans la quatrième partie du roman, le jeune lieutenant romain, tout juste âgé de vingt-deux ans, est envoyé en compagnie de onze vaillants chevaliers et à la tête d'une puissante armée reconquérir les terres que Rome a perdues durant ses démêlés avec Carthage. Sur le conseil de Luces, un Romain qui avait été autrefois l'amant de la reine de Grande-Bretagne, Cerse, elle-même une Romaine, César décide d'attaquer la Grande-Bretagne et

\footnotetext{
${ }^{15}$ Voir mon article, «Conquering Arthurian Romance : Alexander in Perceforest», The Medieval French Alexander, éd. Donald Maddox et Sara Sturm-Maddox, Albany (New York), SUNY Press, 2002, p. 203-217.

${ }^{16}$ Dans la troisième partie du roman (Perceforest, troisième partie, tome 1, éd. G. Roussineau, Genève, Droz, 1988, paragraphe XXIX, p. 357-382 ), il est raconté comment les Romains débarquent dans les îles britanniques, en Ecosse, conduits par Julicès, fils d'un sénateur romain, que Lyonnel, lieutenant du roi Gadiffer d'Ecosse, affronte en combat singulier. Vainqueur, Lyonnel assure ainsi la victoire des Ecossais et sauve le royaume.

${ }^{17}$ Sur ce point, voir l'article de J.H.M. Taylor, «The Fourteenth Century : Context, Text and Intertext ", The Legacy of Chrétien de Troyes, ed. N. Lacy, D. Kelly et K. Busby, Amsterdam, Rodopi, vol. 1, p. 267-332.
} 
l'Ecosse et de venger ainsi la sévère défaite que les Romains s'étaient vu infliger des années auparavant. Aidés par la trahison de Cerse, César et ses hommes mettent la Grande-Bretagne à feu et à sang. Une grande et ultime bataille se déroule auprès du Franc Palais, la résidence fondée par le roi Perceforest. Tous les chevaliers bretons et écossais y trouvent la mort. Ainsi César mène-t-il à bien la conquête de la GrandeBretagne, non sans subir toutefois de lourdes pertes. Dans sa lancée, il conquiert la Gaule. Momentanément tenu en échec devant la cité de Nerves (Tournai) avec laquelle les Romains sont en guerre depuis des décennies ${ }^{18}$, César revient en force et anéantit la ville, assurant ainsi la domination de Rome sur le Nord et l'Ouest de l'Europe ${ }^{19}$. Dans l'ensemble de l'épisode et en particulier durant la bataille du Franc Palais, César est présenté comme un remarquable chef de guerre: preux, comme le prouve son corps à corps avec le Chevalier Doré, sage et prudent : il écoute les bons conseils de son entourage ; magnanime : pris de compassion devant les souffrances des chevaliers bretons, il tente de les convaincre de cesser un combat inutile et déplore ensuite leur mort. Mais animé par son désir de gloire, il ne peut renoncer à la conquête quel qu'en soit le prix : non seulement il détruit toutes les villes de GrandeBretagne, ravage le pays et en décime la population, mais encore il n'épargne pas sa propre armée: plus de 10600 Romains ont trouvé la mort lors de la bataille du Franc Palais ${ }^{20}$ au point que le chef romain doit momentanément renoncer à attaquer Nerves, vu la faiblesse de ses effectifs. On le voit, bien que confronté à des personnages de roman, César conserve les traits du personnage historique. On pourrait même arguer qu'avec son intervention le Roman de Perceforest prend une orientation nouvelle ou plutôt retrouve l'orientation de son $\operatorname{prologue}^{21}$ : il se fait chronique, comme si César l'attirait dans le champ historique.

Un épisode toutefois modifie curieusement l'image du personnage historique et, l'espace de quelques folios, donne à César les traits d'un personnage de roman : il s'agit du récit de sa mort. L'empereur est en effet assassiné par les descendants du roi Perceforest qui entendent venger leurs ancêtres. Perceforest reprend quelques détails historiques (ou pseudo-historiques) bien connus : l'arme du crime, le rôle des sénateurs, le personnage de Brutus, et les intègre habilement dans sa réécriture de l'épisode dont l'authenticité se trouve ainsi garantie. Mais il développe aussi considérablement l'événement en en proposant une nouvelle préhistoire. Le cas de l'objet avec lequel est perpétré le meurtre est de ce point de vue significatif. Dans Li Fet des Romains par exemple, il est simplement signalé que les conjurateurs frappent César de leurs greffes ${ }^{22}$. Dans le Perceforest, l'arme du crime a une origine lointaine

\footnotetext{
${ }^{18}$ Sur l'origine de cette guerre, voir Perceforest, quatrième partie, tome 1, paragraphe XIX, p. 412-416, éd. G. Roussineau, Genève, Droz, 1987.

${ }^{19}$ Toute cette conquête est racontée dans la quatrième partie du roman, tome 1, paragraphes XXVII à XXIX

${ }^{20}$ Quatrième partie, tome 1, p. 647.

${ }^{21}$ Sur ce point, voir mon article «Le clerc et le ménestrel : prose historique et discours versifié dans le Perceforest », Cahiers de Recherches Médiévales, numéro 5, Ecrire en prose (XIII ${ }^{e}$ $X V^{e}$ siècles) : histoire et fiction, 1998, p. 87-105.

${ }^{22}$ Voir par exemple: «Cassius le feri de son grefe en la gorge et li fist une petite plaie (...). Brutus le feri dou sien grefe el piz (éd. L.F. Flutre et K. Sneyders de Vogel, Paris-Groningue : Droz-J.B. Wolters, 1938, p. 740). La mort de César est racontée en sept pages (enterrement
} 
et complexe : lors de la bataille du Franc Palais, Jules César a frappé à mort Nestor, le fils cadet de Gadiffer et de la reine fée. Retirant le fer de lance de la plaie de son fils, la reine s'exclame :

«Rommains, mauvaises gens, maint pays avez destruit et maint homme mis a mort. Et au regard de moy je m'en plains pour mes deux filz et pour mes aultres amis, et par especial de Julius Cesar qui a esté chief de ceste guerre et qui a occis mon chier filz de ce fer duquel il le couvendra une fois morir. Si vive tant comme il pourra, mais a celle fin le couvendra il venir. »(Perceforest, Ars. 3491, fol. $45^{\mathrm{r}}$ ).

La prophétie de la reine va s'avérer mais non sans que le fer de lance connaisse toutes sortes d'aventures, de transformations et de transferts. La reine, qui s'est retirée avec son époux et son beau-frère Perceforest sur l'Ile de Vie, le conserve avec soin pendant les nombreuses années où la Grande-Bretagne et le lignage de Perceforest se remettent difficilement de la terrible défaite du Franc Palais. Puis elle le donne à Ourseau, son petit-fils, romain de par sa mère, et lui confie la mission de venger ses oncles et son grand-père sur César. Ourseau garde religieusement le fer de lance et se prépare à retourner à Rome. Mais sa femme, redoutant son départ, vole le fer de lance dont elle fait faire «douze greffes pour ouvrer de soie » (Perceforest, Ars. 3491, fol. 45 ${ }^{\mathrm{r}}$ ). Ourseau, averti par un songe de l'entreprise de sa femme, récupère les greffes et se rend à Rome. En croyant détourner son mari de son projet, la femme d'Ourseau n'a fait que participer à l'accomplissement de la prophétie de la reine-fée. Les douze greffes, destinées à l'origine aux travaux d'aiguille des femmes et reconverties en stylets, instruments d'écriture, vont venger à la fois les chevaliers tombés lors de la bataille du Franc Palais et leurs veuves. En effet elles vont s'avérer les armes parfaites pour le meurtre de l'empereur qui sera perpétré par les «douze plus notables » de la cité (Perceforest, Ars. 3491, 51 $1^{\mathrm{r}}{ }^{23}$. Très fines, elles s'enfoncent dans la poitrine de César sans faire jaillir la moindre goutte de sang, ce qui permettra aux meurtriers de l'empereur d'affirmer que celui-ci s'est simplement écroulé devant eux, victime d'un soudain malaise. Loin d'être inquiétés comme ils le sont par exemple dans Li Fet des Romains ${ }^{24}$, les conjurateurs peuvent au contraire feindre la surprise et l'innocence ${ }^{25}$.

La mise en place de la conjuration est également présentée de manière nouvelle, car il s'agit de faire jouer un rôle de premier plan aux descendants de Percefo-

compris) dans Li Fet (p. 738-744). On comparera aux treize folios (ms Ars. fr. 3491 fol. 41-

54) consacrés à l'épisode dans le Perceforest.

${ }^{23}$ Dans Li Fet, plus de soixante sénateurs trempent dans la conjuration, les chefs étant cependant Cassius et Brutus. César est frappé de vingt-trois coups (ou vingt-quatre selon la manière dont ils sont dénombrés) dont seul celui porté par Brutus est mortel.

${ }^{24}$ Où il est dit que certains des conjurateurs se suicident avec les «grefes meïsme $<\mathrm{s}>$ dont il orent Cesar ocis » (p. 744).

${ }^{25}$ Quand les gens de l'empereur pénètrent dans le Capitole et trouvent leur maître mort, le chef des conjurateurs, Ursus Bouce Suave, leur déclare : «l'empereur qui estoit venu par devers nous pour avoir nostre advis et conseil sur ce signe [le présage inquiétant qui a motivé la venue de l'empereur] en a moustré en nostre presence l'exposition ainsi que vous povez veoir car tantost qu'il eut racompté l'adventure de ce signe, il est cheu mort soubdainement devant nous tous. »(Perceforest, Ars. fr 3491, fol. 54 ${ }^{\mathrm{r}}$ ). 
rest. Rentrant à Rome avec les douze greffes, Ourseau retrouve son vieux père, sénateur romain, et ses frères parmi lesquels Ursus Bouce Suave, ainsi nommé parce que «tous ses parlers sambloient estre auctoritez et par son sens et son bel langaige il estoit doien de Capitole, car tous les jugemens et les arrestz estoient pronunciez de sa bouce» (Perceforest, Ars. 3491, fol. $47^{\mathrm{r}}$ ). Ursus Bouce Suave prend l'initiative d'organiser la vengeance. Il conseille d'abord d'isoler César en détournant de lui les hommes sur lesquels il fonde sa force, puis d'attendre le signe qui, selon une prophétie formulée par le luiton Zéphyr, marquera le changement de fortune de César. Il recommande aussi de mettre par écrit le récit de son frère Ourseau et les préparatifs de la vengeance afin que l'histoire reste en mémoire.

Le temps passe, César devient de plus en plus puissant. Le Perceforest, tressant habilement événements historiques et événements fictionnels, décrit ainsi sa spectaculaire ascension et la haine qu'il continue de nourrir à l'égard des habitants de la Grande-Bretagne :

«Julius Cesar monta depuis en si grant honneur qu'il fu fait empereur de Romme ; mais anchois fu envoié en Gaule et quant il eut conquis tout le paiis, il sceut que le roiaulme de Bretaigne restoit en bon estat et a ce temps en estoit roy Cassibelamus. Et pource qu'il ne rendoit point de treu aux Rommains, Julius Cesar en eut grant despit et de fait ala sur lui a groz navire pour conquerre le paiis. Mais il l'en couvint retourner honteusement, car il trouva Cassibelamus tres vaillant et fort garny de bonne chevalerie par tout son paiis. Mais depuis Cassibelamus eut discord a l'encontre de Androgeo duc de Trinovant que nous nommons a present Londres. Cestuy Androgeo manda Julius Cesar qui se tenoit en Gaule, et quant il fu descendu en Bretaigne, eulx deux firent tant que Cassibelamus fu submis a la cité de Romme, ou il fist tant assez tost aprez par sa puissance qu'il mist a mort Pompee son serourge et encassa le sage Caton morir en Libe. Et aprés ce il conduisi telement son fait qu'il fut empereur de Romme tousjours demourant la haine sur lui es courages de celx qui estoient descendus du bon lignaige au roy Perceforest, ainsi que dit est. » (Perceforest, Ars. fr 3491, fol. $48^{\mathrm{r}}$ )

Ce passage résume, avec une certaine liberté, les deux expéditions de César en Grande-Bretagne telles qu'elles sont rapportées dans la partie consacrée à la guerre des Gaules dans Li Fet des Romains ${ }^{26}$. Pompée et Caton ne sont pas totalement oubliés, mais ils jouent un rôle modeste: ils ne sont que quelques-unes des victimes de la tyrannie de César. On apprend en effet un peu plus tard que plus de deux cent mille Romains ont trouvé la mort à cause de César. Ce chiffre rappelle «la grant effusion de sang [...et] terrible desconfiture du lignaige humain » causées par la bataille de Pharsale, dont le Jouvencel pour sa part, on s'en souvient, rejette la responsabilité sur Pompée, et que Li Fet des Romains déplorent aussi de leur côté ${ }^{27}$. Si Perceforest réutilise des éléments historiques, on voit néanmoins qu'il met toujours au premier plan la lutte entre le peuple britannique et Jules César.

\footnotetext{
${ }^{26}$ Voir les chapitres X et XI de la partie II dans l'édition Flutre et Sneyders de Vogel, p. 166190.

${ }^{27}$ L'épisode de la bataille de Pharsale est raconté en III, 12, p. 511-544. Voir la déclaration du narrateur p. 537 : «Si granz ocisions de gent n'avoit esté en une place des le conmencement dou monde, <ne > ne fu apres. »
} 
Alors que la fortune sourit à l'empereur, le vieux sénateur romain meurt, ses fils, Ourseau, Ursus Bouce Suave et leurs frères vieillissent, mais la relève est assurée par un fils d'Ursus, également nommé Ursus Bouce Suave qui va devenir le chef des conspirateurs. Se souvenant toutefois de l'existence de Brutus, Perceforest introduit le personnage dont il fait un cousin du côté maternel du jeune Ursus Bouce Suave. Quand les signes annonciateurs du déclin de César se manifestent, Brutus offre sa collaboration à Ursus et se déclare prêt à frapper le premier pour venger son propre père mis à mort par l'empereur. Le meurtre de César devient donc une complexe affaire de famille et si de grandes causes comme la liberté des citoyens romains ou la lutte contre la tyrannie sont alléguées à plusieurs reprises ${ }^{28}$, on reste avec le sentiment qu'il s'agit avant tout de venger un lignage particulier ${ }^{29}$. En réduisant de cette manière la portée du meurtre de César, Perceforest «acclimate » le personnage historique au roman.

Paradoxalement toutefois, il va aussi redonner à l'empereur sa stature de personnage exemplaire au moment où il fait le récit du meurtre à proprement parler. La scène se déroule donc dans le Capitole ${ }^{30}$. César vient d'y pénétrer, seul, dans l'intention de demander aux sénateurs de convoquer des astrologues susceptibles de lui expliquer un effrayant présage qui s'est produit la nuit précédente : les fenêtres et les portes de sa résidence se sont soudain mises à claquer violemment. Or le luiton Zéphyr avait prédit que :

«[au] departement [de la bonne fortune de César] les huis et les fenestres de son palais menront telle noise de clore et d'ouvrir que toute Romme en sera espoventee et mesmes Julius Cesar en sera tant esbahy que pour en avoir conseil ira lendemain en chapitre $»\left(\text { Perceforest, Ars. 3491, fol. } 46^{\mathrm{v}}\right)^{31}$.

Le détail se trouve chez Salluste et dans Li Fet des Romains dont s'inspire Perceforest pour l'ensemble de l'épisode ${ }^{32}$. Mais ce qui lui donne un sens nouveau,

\footnotetext{
${ }^{28}$ Brutus déclare par exemple à Ursus : «Je ne puis croire que si tressoubdaine haultesse ne doive cheoir a coup ne que le pechié qu'il a commis a cause du poeuple qu'il a fait mettre a mort en acquerant l'onneur dont il jouist a present, qui monte jusques a deux cens mil hommes avecques la francise de la cité qu'il a par sa force et cruaulté abatue, demeure a pugnir, parquoy sa haultesse declinera soubdainement » (Perceforest, Ars. 3491, fol. $49^{v}$ ).

${ }^{29}$ Les termes par lesquels Ursus condamne César à mort montrent combien au bout du compte, l'aspect «vendetta » l'emporte sur la logique politique, pourtant présentée d'abord : «Consideré le fait de Julius qui est icy present, lequel a esté a l'encontre du bien commun, l'onneur et la francise de la noble cité de Romme et l'admendrissement des nobles hommes du paiis, car il n'y a icy personne a qui Julius que cy est n'ait fait mettre a mort ou son pere ou ses freres ou ses amis charnelz, pour laquelle cruaulté, je le juge a morir par les douze greffes que nous tenons dont il soit feru tant que l'ame luy soit hors du corps. Et par ainsi sera acomplie la prophecie de la roine faee et du sage Zephyr et vengance prinse des preudommes de la Grant Bretaigne et de noz autres amis qu'il mist et fist mettre a mort » (Perceforest, Ars. 3491, fol. $51^{\mathrm{r}}-52^{\mathrm{v}}$ ).

${ }^{30}$ Dans Li Fet des Romains, le meurtre a lieu dans la Cort Pompee.

${ }^{31}$ Voir aussi quatrième partie, tome 2, paragraphe LVII, p. 1098.

${ }^{32}$ Ce présage, ainsi que d'autres, est rapporté par Li Fet des Romains p. 739. Toutefois, au lieu de pousser César à se rendre au sénat dans l'espoir de se le faire interpréter, le signe réussit presque à l'en détourner.
} 
c'est que le signe s'est déjà produit au Franc Palais, résidence de Perceforest, à l'occasion de l'institution de la table du Franc Palais, ancêtre de la Table Ronde ${ }^{33}$. Le même présage annonce ainsi la naissance d'une chevalerie d'élite et la mort de celui qui en a causé la destruction.

Les conspirateurs du Capitole en comprennent évidemment sans difficulté le sens. Il n'en va pas de même pour César qui pourtant avait reçu un avertissement, un billet lui recommandant de ne pas se rendre au Capitole ce jour-là ${ }^{34}$. Mais l'empereur n'a pas pris connaissance du message: Ursus découvrira le billet, encore scellé, serré dans le poing de l'empereur mort. Ignorant du sort qui l'attend, César se présente donc devant les sénateurs et leur expose la raison de sa venue. Ursus prend alors la parole pour lui expliquer lui-même le présage et lui reprocher sa tyrannie. Puis il invite ses compagnons à inscrire sur leurs tablettes le jugement qu'ils réservent à César et à cet effet, il distribue les douze greffes. À ce moment du récit, le narrateur prend la parole et, s'adressant directement aux lecteurs, tire la leçon du drame qui est en train de se jouer :

En verité, beausseigneurs, c'est une merveilleuse chose a consciderer l'estat du monde, car ung homme sera a ung temps si courageuz, si fier, si constant et si sage de soy gouverner, car pour paroles fieres, terribles et plaines de menaces ne pour anemis presens, garnis de fiereté et grant orgueil, sans entendre a mercy, il ne se meult, ainchois demeure en son sens et en son hardement tresaffecté de soy deffendre telement qu'il convaint ses ennemis, ja soient ilz plus. Et une autre heure, il lui est tant contraire que a l'encontre de mendre et non puissant de lui, il n'a courage ne hardement de soy deffendre, ainchois meurt honteusement. J'ay racompté cest example pour ce que a ce point, c'estoit une chose merveilleuse et piteuse a estre ou Capitoile, car Julius Cesar l'empereur estoit devant les seigneurs de la cité que lui mesmes avoit creez et faiz et qui par avant avoit esté si entreprenant, si preu, si chevallereuz et si conquerant qu'a petite compaignie de gens eust francement atendu le remanant des hommes du monde en bataille. (Perceforest, Ars. 3491, fol. $51^{\mathrm{v}}-52^{\mathrm{r}}$ )

Dans ce discours, César devient donc la malheureuse victime de l'instabilité de Fortune et son cas illustre le proverbe bien connu : «Qui plus haut monte qu'il ne doit, de plus haut chet qu'il ne voudroit $»^{35}$. Si le proverbe n'apparaît pas sous cette

${ }^{33}$ Roman de Perceforest, deuxième partie, vol. II, éd. G. Roussineau, Genève, Droz, 2001, p. 245 : «ilz oïrent clorre les fenestres du palais toutes a une fois si roid que c'estoit merveilles a oÿr. Et quant le roy et tous ceulx des tables oörent la noyse, ilz eurent merveilles que ce povoit estre a dire. Sy prindrent a regarder amont, mais ilz n'eurent pas longuement regardé quant les fenestres alerent toutes ouvrir. » Une manifestation assez similaire se produit également à l'arrivée de Galaad au début de la Queste del Saint Graal: portes et fenêtres se ferment d'elles-mêmes (Queste del Saint Graal, éd. A. Pauphilet, Paris, Champion, rééd. 1978, p. 7).

${ }^{34}$ «Franc empereur, deporte toy pour meshuy d'aler ou Capitoile, car ta mort y gist, mais demain fay ton vouloir» (Perceforest, Ars. 3491, fol. 52 $2^{v}$ ). Dans Li Fet des Romains, sur le chemin de la Cort Pompee, César rencontre un homme qui lui remet un billet lui révélant la conjuration. Mais César le met dans sa main gauche avec toute une série d'autres documents qu'il compte lire plus tard (p. 740).

${ }^{35}$ Voir proverbe M176 dans J. W. Hassel, Middle French Proverbs, Sentences and Proverbial Phrases, Toronto, Pontifical Institute of Mediaeval Studies, 1982. 
forme dans le passage, on en trouve cependant une variante: «qui est plus hault monté et plus se quassera au cheoir»(Perceforest, Ars. 3491, fol. $48^{\mathrm{v}}$ ). Mais il y a plus et plus surprenant. Alors que dans tout l'épisode, César a été condamné pour sa soif de pouvoir et sa cruauté, voilà que le narrateur le présente comme un modèle de bon prince :

Quy se teniste dont de soy esmerveillier et de avoir trés grant pitié qui a ce point eust esté en la presence des juges ou Capitoile et regardast a plain le plus conquerant, le plus preu, le plus hardy de son temps et le plus sage, le plus courtois, le plus misericordieux au priant mercy qui fust en son regne et qui estoit tel prince que a lui obeissoient toutes seignouries et tous les hommes du monde sinon tant seulement douze devant lesquelz il se retrouva fort esbahy? (Perceforest, Ars. 3491, fol. 52 $2^{\mathrm{r}}$ (36 $^{36}$

Le plus paradoxal est que ce revirement, qui rappelle la manière contradictoire dont le Jouvencel traite l'exemple de César, est le fait non seulement du narrateur mais encore du personnage d'Ursus Bouce Suave lui-même. Devant le corps de l'empereur, alors même qu'il ne se trouve qu'en compagnie des conspirateurs, Ursus prononce en effet un émouvant panégyrique :

Ha a! Tres noble prince sage, courtois, large, vaillant, chevalereuz, conquerant sur les rebelles, piteuz et pardonnant a ceulx qui requeroient mercy, amoderé en tes fais que tu as conduitz par grant prudence et discretion, car tu ne meis onques ne feis mettre homme a mort en ton courouz ou sans cause. Ha a! Fleur et le mireoir de toute chevalerie! Ha a! Clareté et lumiere pour tous princes et seigneurs subgetz a toy donner a congnoistre le droit chemin de gouverner. Ha a! Prince souffissant et le chief et empereur de tout le monde, que t'est il advenu? (Perceforest, Ars. 3491, fol. $53^{\mathrm{r}}$ ).

Comment comprendre cette apparente contradiction? Il me semble qu'elle trahit un brusque changement de statut du personnage. Une fois mort en effet, César échappe au roman, il retrouve sa dimension historique et redevient le grand héros antique, comparable à Alexandre. Du reste, l'éloge funèbre que lui décerne Ursus rappelle celui qu'a suscité l'annonce de la mort d'Alexandre ${ }^{37}$. Le revirement final me paraît procéder de la volonté de rétablir in extremis une image plus nuancée de César, de rendre au personnage historique la part de gloire et d'honneur qui lui revient $^{38}$. Dans le Perceforest, César, comme Alexandre ${ }^{39}$, suscite des sentiments partagés qui font écho à ceux qu'éprouvent les écrivains du Moyen Âge d'une manière générale devant les héros antiques : un mélange d'admiration et une certaine répul-

\footnotetext{
${ }^{36}$ Voir aussi : «Julius Cesar le gentil empereur qui estoit digne d'avoir tout le gouvernement du monde » (Perceforest, Ars. 3491, fol.52 ${ }^{\mathrm{v}}$ )

${ }^{37}$ Voir Perceforest, deuxième partie, tome 1, éd. G. Roussineau, Genève, Droz, 1999, p. 102.

${ }^{38}$ Sur l'ambivalence de l'auteur des Fets des Romains face à César, voir C. Croizy-Naquet, Écrire l'histoire romaine au début du XIII e siècle, Paris, Champion, 1999, en particulier p. 244-249.

${ }^{39}$ Cette ambivalence est très nette en ce qui concerne Alexandre. Sur ce point, voir mon article «Conquering Alexander», art.cit.
} 
sion pour de grands hommes dont ils ne peuvent évidemment ni embrasser le paganisme ni louer la soif de pouvoir personnel.

Dans le Jouvencel, on l'a vu, l'exemple de César sert à illustrer une vérité générale présentée préalablement. Dans le Perceforest, à l'inverse, l'auteur déduit une vérité générale du cas particulier de César. Mais le résultat est le même : la mort de César illustre l'instabilité de Fortune. Dans l'un et l'autre cas, César est une figure exemplaire. S'il a pu pour un temps devenir un personnage romanesque, c'est parce que sa trajectoire s'est trouvée aimantée par celle des héros du roman. Mais sa mort, aux mains des sénateurs romains, le libère du lien qui l'unissait au lignage de Perceforest, d'où le fait qu'Ursus peut lui reconnaître une valeur qu'il lui déniait de son vivant. La vengeance une fois accomplie, les meurtriers et le cadavre disparaissent totalement de l'histoire. Paradoxalement le meurtre n'entraîne aucune conséquence, ni faste, ni néfaste, pour le lignage de Perceforest. Alors que malgré les phases de destruction et de restauration qui se succèdent en Grande-Bretagne, l'œuvre d'Alexandre persiste, que son influence se fait toujours sentir bien au-delà de sa mort, rapportée dans le second livre, l'intervention de César dans le roman apparait finalement comme un simple accident de l'histoire, comme si la collision de sa trajectoire avec celle du monde arthurien n'avait été qu'un caprice de Fortune corrigé par un autre.

Au terme de cette analyse, il me faut apporter une réponse à la question que j'ai posée. César est-il un personnage de roman? Il me semble que non et que la tentative du Perceforest, toute intéressante qu'elle soit, le prouve. Malgré l'habileté avec laquelle ce roman réécrit l'épisode du meurtre, il ne parvient pas à présenter César autrement qu'un grand conquérant perdu par son orgueil et son ambition, c'est-à-dire comme un personnage exemplaire. Pourquoi César résiste-t-il à tout «enromancement»? Peut-être parce que le texte principal qui en retrace la biographie, Li Fet des Romains, sur lequel s'appuient aussi bien Jean de Thuin que l'auteur du Perceforest, est un texte à vocation historique. Sans doute aussi parce que, à la différence d'Alexandre dont l'ambition était à la fois militaire et intellectuelle, César n'apparaît pas comme engagé dans une quête du savoir. C'est un homme de guerre. On peut aussi se demander si le fait qu'il soit lui-même un historien, dont l'œuvre a été connue et citée dès le haut Moyen Âge, ne lui a pas fermé l'accès au roman qui, quoique flirtant avec l'histoire (surtout au $\mathrm{XV}^{\mathrm{e}}$ siècle), n'est pas parvenu cependant à plier à ses fins un personnage pour le coup trop historique !

Michelle Szkilnik Paris III 